\title{
The Tradeoff between Mean Delay and Energy Saving Factor under DRX Scheme
}

\author{
Mohammad Asif \\ Hossain \\ Department of Computer \\ Systems and Technology \\ University of Malaya, \\ Kuala Lumpur, Malaysia
}

\author{
Md. Imdadul Islam \\ Department of Computer \\ Science and Engineering \\ Jahangirnagar University, \\ Savar, Dhaka, \\ Bangladesh
}

\author{
Fahima Tabassum \\ Institute of Information \\ Technology, \\ Jahangirnagar University, \\ Savar, Dhaka, \\ Bangladesh
}

\author{
M. R. Amin \\ Department of \\ Mathematical and \\ Physical Sciences, \\ East West University, \\ Dhaka, Bangladesh
}

\begin{abstract}
Recently power saving is a vital issue for wireless devices of $4 \mathrm{G}$ and $5 \mathrm{G}$ networks. A device enters in sleeping mode (short and long sleep cycle) when there is no arrival of traffic but wakeup once the arrival of traffic. Before wakeup, the UE user equipment (UE) spends the rest of the sleeping cycle which incurs a delay of service. There is a tradeoff between the length of a sleep cycle (power saving factor ids higher for longer sleep cycle) and mean delay of service. In this paper, a Markov chain is designed including timer inactivity, short sleep, and long sleep and active service states. The closedform solution of the chain is performed using node equations hence comparison of performance is made with previous work in the context of power-saving factor and mean delay. Both the power saving factor and mean delay of this paper are found marginally better than the previous work at lower packet arrival rate but at higher arrival rate performance are almost the same but claims some explanations.
\end{abstract}

\section{General Terms}

Energy savings, wireless communication, Long Term Evaluation.

\section{Keywords}

LTE, DRX, long sleep, short sleep, QoS, Markov Chain.

\section{INTRODUCTION}

The data rate of the Long Term Evaluation (LTE) network has increased tremendously compared to the previous 3G network. LTE provides many services like mobile TV, realtime gaming, HD video streaming and so on. However, these services consume the battery life of the devices very rapidly. The size of the battery is not increased to cope with the data rate. For this reason, several methods have been implemented to reduce the energy consumption of UE in wireless networks including idle/sleep modes in WiMAX and discontinuous reception (DRX) in LTE summarized in [1].

The DRX mechanism of LTE is a power-saving mechanism that saves the UE's battery. When there is no data to receive by the UE from the evolved Node-B (eNB), UE will go for short sleep mode, then wake up periodically at pre-determined times to check whether there is any data to receive or not. After a certain period of short sleep, UE will go for a long sleep. In these sleeping modes, UE will turn off its circuitry related to the transmission-reception to save the battery life found in [2]. However, there is a tradeoff between this power saving mechanism and user-end delay. For example, eNB has some data to transmit to the UE, but the UE is in sleeping mode, therefore it will wake up after its pre-determined schedule. In this case, the eNB has to wait until the UE wakes up, hence the communication system will experience a delay. This delay will hamper the applications which are delaysensitive [3]. Intense research work is going on regarding the delay or latency of the wireless network. In [4], the authors have proposed a fuzzy-based power-saving scheduling scheme for IoT over the LTE/LTE-A networks. They have focused on three individual metrics: radio resource management, power consumption and the unexpected delay caused by the DRX mechanism from the scheduling and resource allocation perspective. The authors made a tradeoff among three individual metrics.

The authors in [5], showed the trade-off between the powersaving and wake-up delay performance based on the analytical model. The paper provides an overview of adjustable and non-adjustable DRX cycles of the LTE/LTE- A power-saving mechanism. They have modeled the system with bursty packet data traffic using a semi-Markov process. Ali et al., in [6] have developed an analytical model to estimate power saving achieved and latency incurred by DRX operation. They have formulated a tradeoff scheme to maintain a balance between these two parameters based on the operator's preference for power saving. They showed that their proposed scheme can achieve a significant delay improvement with a small decrease in power saving. Their results have indicated that the short DRX cycles are very effective in reducing latency for active traffic. They have pointed out the necessity of optimizing DRX configuration in order to maintain a tradeoff between power saving and buffering delay. Their conclusion is that a higher number of short DRX cycles is preferable for active traffic. The impact of latency on the power saving factor is also shown in [7-12].

In the previous work of the authors of this paper in [13], the sleep mode of the LTE network has been analyzed based on two statistical models: Poisson's probability density function (pdf) and Engset pdf with the help of Markov chain. In [14], the state transition chain contains three states: serving the state, state of timer inactivity and silent state, where a simplified statistical model using traffic parameters of arrival rate, pdf of interarrival time and its threshold value have been considered. In [15], a new simplified model of two states was developed. The model is helpful to measure the inclination of sleep and active state of a UE on the LTE network using the entropy of binary state. In [16] the concept of DRX is explained in a different way called optimistic DRX (ODRX), which is modeled by the finite state machine. The profiles of a power-saving factor against wakeup latency, data arrival rate and length of the cycle are shown with a comparison of DRX. A similar concept is available in [17], where the state transition chain is developed using a semi-Markov model and the results deal with the same parameters. Five steps Markov 
chain of the energy-saving model is found in [18] for 5G user equipment (UE). This paper mainly deals with analytical results on a state transition chain of ten states for the DRX scheme. The relation between traffic parameters, latency, and power-saving factors are shown graphically with some comparison of previous work.

This paper is organized as section 2 deals with the DRX scheme of UE using Markov chain with provides a complete solution of the chain and derivation of all parameters of DRX, section 3 provides the discussions of the results obtained based on a statistical analysis of section 2 and section 4 concludes the entire analysis.

\section{SYSTEM MODEL}

A UE under DRX enters a sleeping state when there is no downlink traffic for it for some duration above the threshold time. The UE wakes up from the sleeping state periodically to check whether it has any downlink packet from eNB. The time duration for which the UE remains in 'on state' for such checking is indicated as $T_{O N}$. The length of $T_{O N}$ is about the duration of a few sub-frame. If there is no incoming packet for the UE within this $T_{O N}$ period, then the UE will go to sleep mode again. When there is any packet destined for UE, then it starts the 'inactivity time' and goes to the continuous reception mode and on an average, it stays on that state for duration of $T_{0}$.

Figure. 1 shows the state transition chain of UE under DRX scheme, consists of 10 states. The state 0 is continuous reception state and states 1 to 9 are sleeping states. The sleep states are divided into two categories: states 1 to 6 are considered as short sleep states and that of 7 to 9 are long sleep states.

Table 1 shows the parameters used in designing the state transition chain are.

Let us apply node equations of [19-21] on node $S_{1}$,

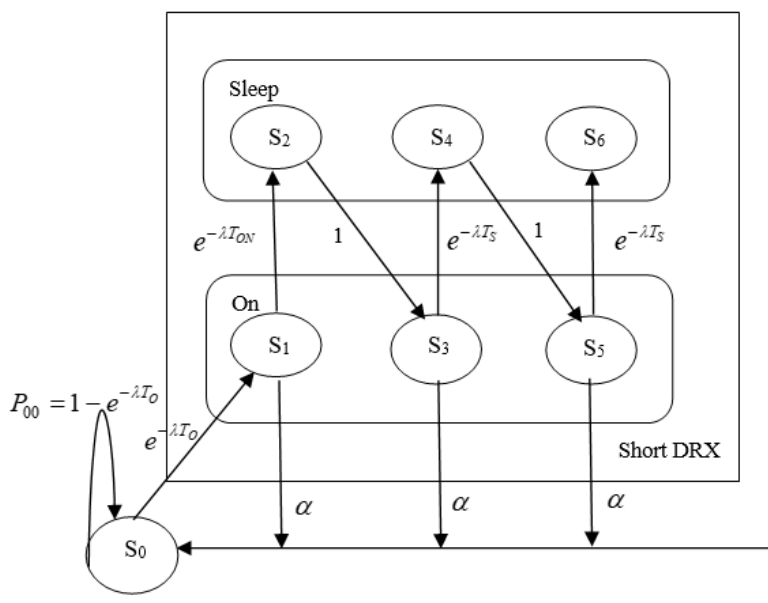

Figure. 1 State Transition between ON mode to short sleep and long sleep mode

$\Rightarrow S_{1}=S_{0} \frac{e^{-\lambda T_{0}}}{\left(e^{-\lambda T_{O N}}+\alpha\right)}$

Node equation on state $S_{2}$,

$$
S_{2} .1=S_{1} e^{-\lambda T_{O N}}=S_{0} \frac{e^{-\lambda\left(T_{0}+T_{O N}\right)}}{\left(e^{-\lambda T_{O N}}+\alpha\right)} .
$$

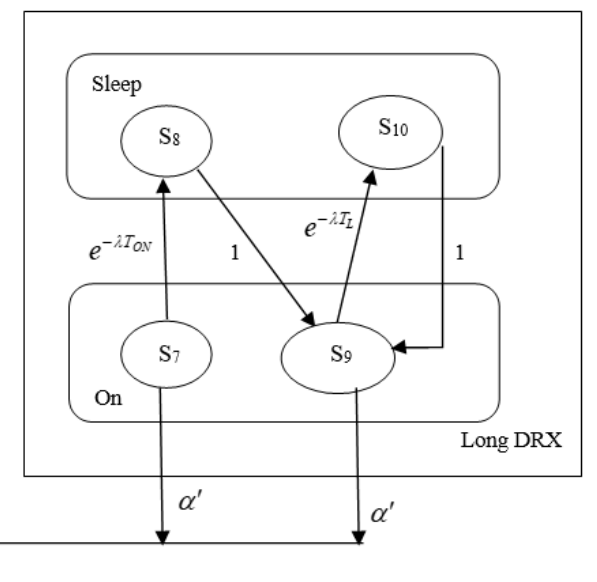

$$
S_{1}\left(e^{-\lambda T_{O N}}+\alpha\right)=S_{0} e^{-\lambda T_{0}}
$$

\begin{tabular}{|c|c|}
\hline Symbol & Meaning \\
\hline$S_{0}$ & $\begin{array}{l}\text { probability of staying in continuous } \\
\text { reception mode }\end{array}$ \\
\hline$S_{i}: i=1$ to 6 & $\begin{array}{l}\text { probability of staying in short sleep } \\
\text { mode }\end{array}$ \\
\hline$S_{i}: i=7$ to 10 & $\begin{array}{c}\text { the probability of staying in long sleep } \\
\text { mode }\end{array}$ \\
\hline$\alpha$ & $\begin{array}{l}\text { probability of transition from short } \\
\text { DRX to continuous reception mode }\end{array}$ \\
\hline$\alpha^{\prime}$ & $\begin{array}{l}\text { probability of transition from long } \\
\text { DRX to continuous reception mode }\end{array}$ \\
\hline$T_{0}$ & $\begin{array}{l}\text { period of staying in continuous } \\
\text { reception mode }\end{array}$ \\
\hline$T_{S}$ & short DRX cycle \\
\hline$T_{L}$ & long DRX cycle \\
\hline$T_{O N}$ & $\begin{array}{c}\text { when a UE is enabled, it wakes up and } \\
\text { stay in on mode for the duration } \mathrm{T}_{\mathrm{ON}} \text { to } \\
\text { check whether any traffic is available } \\
\text { for it or not }\end{array}$ \\
\hline$\lambda$ & mean packet arrival rate \\
\hline$\lambda_{p c}$ & mean packet arrival rate per session \\
\hline$\mu_{p c}$ & mean packet call length per session \\
\hline$t_{N}$ & $\begin{array}{l}\text { short sleep period (when terminates } \\
\text { from short DRX) }\end{array}$ \\
\hline
\end{tabular}

Table 1: Description of the notations used in the equations.

At $S_{3}$,

$$
\begin{aligned}
& S_{3}\left(\alpha+e^{-\lambda T_{S}}\right)=S_{2} .1 \\
& \Rightarrow S_{3}=\frac{S_{2}}{\left(\alpha+e^{-\lambda T_{S}}\right)}=S_{0} \frac{e^{-\lambda\left(T_{O}+T_{O N}\right)}}{\left(e^{-\lambda T_{S}}+\alpha\right)\left(e^{-\lambda T_{O N}}+\alpha\right)} .
\end{aligned}
$$

At $S_{4}$, 
$S_{4}(1)=S_{3} e^{-\lambda T_{S}}$

$\Rightarrow S_{4}=S_{0} \frac{e^{-\lambda\left(T_{O}+T_{O N}+T_{S}\right)}}{\left(e^{-\lambda T_{S}}+\alpha\right)\left(e^{-\lambda T_{O N}}+\alpha\right)}$.

At $S_{5}$,

$S_{5}\left(\alpha+e^{-\lambda T_{S}}\right)=S_{4} \Rightarrow S_{5}=S_{0} \frac{e^{-\lambda\left(T_{O}+T_{O N}+T_{S}\right)}}{\left(e^{-\lambda T_{O N}}+\alpha\right)\left(e^{-\lambda T_{S}}+\alpha\right)^{2}}$

At $S_{6}$,

$S_{6} \times 1=S_{5} e^{-\lambda T_{S}}$

$\Rightarrow S_{6}=S_{0} \frac{e^{-\lambda\left(T_{O}+T_{O N}+2 T_{S}\right)}}{\left(e^{-\lambda T_{O N}}+\alpha\right)\left(e^{-\lambda T_{S}}+\alpha\right)^{2}}$.

At $S_{7}$,

$S_{7}\left(\alpha^{\prime}+e^{-\lambda T_{S}}\right)=S_{6}$

$\Rightarrow S_{7}=S_{0} \frac{e^{-\lambda\left(T_{O}+T_{O N}+2 T_{S}\right)}}{\left(e^{-\lambda T_{O N}}+\alpha\right)\left(e^{-\lambda T_{S}}+\alpha\right)^{2}\left(e^{-\lambda T_{S}}+\alpha^{\prime}\right)}$.

At $S_{8}$,

$S_{8} \times 1=S_{7} e^{-\lambda T_{S}}$

$\Rightarrow S_{8}=S_{0} \frac{e^{-\lambda\left(T_{O}+T_{O N}+3 T_{S}\right)}}{\left(e^{-\lambda T_{O N}}+\alpha\right)\left(e^{-\lambda T_{S}}+\alpha\right)^{2}\left(e^{-\lambda T_{S}}+\alpha^{\prime}\right)}$.

At $S_{9}$,

$S_{9}\left(\alpha^{\prime}+e^{-\lambda T_{L}}\right)=S_{8} \times 1+S_{10} \times 1$

$\Rightarrow S_{9}=\frac{S_{8}}{\left(\alpha^{\prime}+e^{-\lambda T_{L}}\right)}+\frac{S_{10}}{\left(\alpha^{\prime}+e^{-\lambda T_{L}}\right)}$.

At $S_{10}$,

$S_{10} \times 1=S_{9} \cdot e^{-\lambda T_{L}}$

$\Rightarrow S_{10}=S_{9} \cdot e^{-\lambda T_{L}}$.

From (9) and (10),

$S_{9}=\frac{S_{8}}{\left(\alpha^{\prime}+e^{-\lambda T_{L}}\right)}+\frac{S_{9} e^{-\lambda T_{L}}}{\left(\alpha^{\prime}+e^{-\lambda T_{L}}\right)}$

$\Rightarrow S_{9}\left(1-\frac{e^{-\lambda T_{L}}}{\left(\alpha^{\prime}+e^{-\lambda T_{L}}\right.}\right)=\frac{S_{8}}{\left(\alpha^{\prime}+e^{-\lambda T_{L}}\right)}$

$\Rightarrow S_{9} \times \frac{\alpha^{\prime}}{\left(\alpha^{\prime}+e^{-\lambda T_{L}}\right)}=\frac{S_{8}}{\left(\alpha^{\prime}+e^{-\lambda T_{L}}\right)}$

$\Rightarrow S_{9}=\frac{S_{8}}{\alpha^{\prime}}$ $\therefore S_{10}=\frac{S_{8}}{\alpha^{\prime}} \cdot e^{-\lambda T_{L}}$.

In this paper, 10 probability states of Markov chain are considered. To get the normalized probability states, the sum of probability of entire sampling space is needed as,

$\sum_{i=1}^{10} S_{i}=1$

From (13), the normalized probability of staying at state 0 will be,

$S_{0}=1 /(A+B+C+D+E+F+G+H+I+J)$

, where

$$
\begin{aligned}
& A=\frac{e^{-\lambda T_{0}}}{\left(e^{-\lambda T_{O N}}+\alpha\right)}, \quad B=\frac{e^{-\lambda\left(T_{0}+T_{O N}\right)}}{\left(e^{-\lambda T_{O N}}+\alpha\right)}, \\
& C=\frac{e^{-\lambda\left(T_{O}+T_{O N}\right)}}{\left(e^{-\lambda T_{S}}+\alpha\right)\left(e^{-\lambda T_{O N}}+\alpha\right)}, \\
& D=\frac{e^{-\lambda\left(T_{O}+T_{O N}+T_{S}\right)}}{\left(e^{-\lambda T_{S}}+\alpha\right)\left(e^{-\lambda T_{O N}}+\alpha\right)}, \\
& E=\frac{e^{-\lambda\left(T_{O}+T_{O N}+T_{S}\right)}}{\left(e^{-\lambda T_{O N}}+\alpha\right)\left(e^{-\lambda T_{S}}+\alpha\right)^{2}}, \\
& F=\frac{e^{-\lambda\left(T_{O}+T_{O N}+2 T_{S}\right)}}{\left(e^{-\lambda T_{O N}}+\alpha\right)\left(e^{-\lambda T_{S}}+\alpha\right)^{2}}, \\
& G=\frac{e^{-\lambda\left(T_{O}+T_{O N}+2 T_{S}\right)}}{\left(e^{-\lambda T_{O N}}+\alpha\right)\left(e^{-\lambda T_{S}}+\alpha\right)^{2}\left(e^{-\lambda T_{S}}+\alpha^{\prime}\right)}, \\
& H=\frac{\left.e^{-\lambda\left(T_{O}+T_{O N}+3 T_{S}\right.}\right)}{\left(e^{-\lambda T_{O N}}+\alpha\right)\left(e^{-\lambda T_{S}}+\alpha\right)^{2}\left(e^{-\lambda T_{S}}+\alpha^{\prime}\right)}, \\
& J=\frac{H}{\alpha^{\prime}} \cdot e^{-\lambda T_{L}} \quad I=\frac{H}{\alpha^{\prime}}
\end{aligned}
$$

The other probability states can be determined from eq. (1)(12).

Two important traffic parameters $\alpha$ and $\alpha^{\prime}$ used in the Markov chain of Figure.1 are expressed like [22] as,

$$
\alpha=\left(1-\frac{1}{\mu_{p c}}\right)\left(1-e^{-\lambda_{p c} t_{N}}\right)+\frac{1}{\mu_{p c}}\left(1-e^{-\lambda_{s} t_{N}}\right)
$$

and

$$
\alpha^{\prime}=\left(1-\frac{1}{\mu_{p c}}\right)\left(1-e^{-\lambda_{p c} t_{d e e p}}\right)+\frac{1}{\mu_{p c}}\left(1-e^{-\lambda_{s} t_{d e e p}}\right)
$$

Now the probability of staying in data reception mode is $S_{0}$. The probability of staying in short DRX cycle is,

$P_{S S}=S_{1}+S_{2}+S_{3}+S_{4}+S_{5}+S_{6}$

The probability of staying in long DRX cycle is,

$P_{L S}=S_{7}+S_{8}+S_{9}+S_{10}$ 
The power saving factor,

$$
P_{s f}=\left(P_{S s} \times T_{S}+P_{L S} \times T_{L}\right) /\left(S_{0} \times T_{0}+P_{S S} \times T_{S}+P_{L S} \times T_{L}\right)
$$

Mean Delay in ms

$D=\left(P_{S S} \times T S+P_{L S} \times T_{L}\right) \times 1000$

The next section will deal with numerical results using eq. (1)-(20) of this section.

\section{RESULTS AND DISCUSSIONS}

Taking the usually used value of traffic parameters like [1]: on duration, $T_{O N}=2 \mathrm{~ms}$, duration of continuous reception, $T_{0}=10$ $\mathrm{ms}$, length of short DRX cycle, $T_{S}=80 \mathrm{~ms}$ and length of long DRX cycle, $T_{L}=320 \mathrm{~ms}$; the following six-bar graph of Figure.2 (a)-(f) of 11 probability states have been obtained. In bar graph state 1 correspond to $S_{0}$, state 2 corresponds to $S_{1}$ and so on. Here mean packet arrival rate is taken as, $\lambda=2,5$, 10, 15, 20 and 25 packets/ms for six different bar graph. The numerical value of the probability of entering short sleep state $P_{S S}$, long sleep state $P_{L S}$, power-saving factor $P_{s f}$ and mean delay $D_{\text {mean }}$ in $\mathrm{ms}$ are shown with the heading of the corresponding bar graph. Comparing six bar graphs, it is found that all the 4 above parameters decrease with an increase in packet arrival rate which is also visualized from Figure.3. At a very low packet arrival rate, the UE will mostly stay in a long sleep state hence probability of entering at short sleep state is very low again when the arrival rate starts to grow then UE will move toward a short sleep state from the long sleep state. The phenomenon is visualized from Figure. 3 for $\lambda=1$ to 5 packets $/ \mathrm{ms}$. Further increase in $\lambda$, the UE will have the trend to move to a busy state hence probability of entering short sleep state decreases beyond the value of $\lambda=5$ packets/ms also found from the same figure.

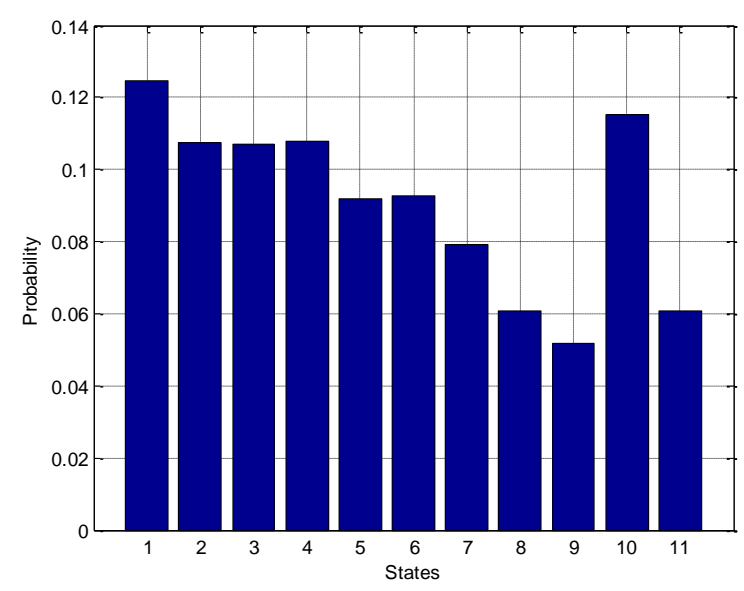

(a) Probability states for $\lambda=2$ packets $/ \mathrm{ms}$,

$$
\begin{aligned}
P_{S S}=0.5867, P_{L S}= & 0.2885, P_{s f}=0.9911 \text { and } D_{M e a n}= \\
& 139.2503 \mathrm{~ms}
\end{aligned}
$$

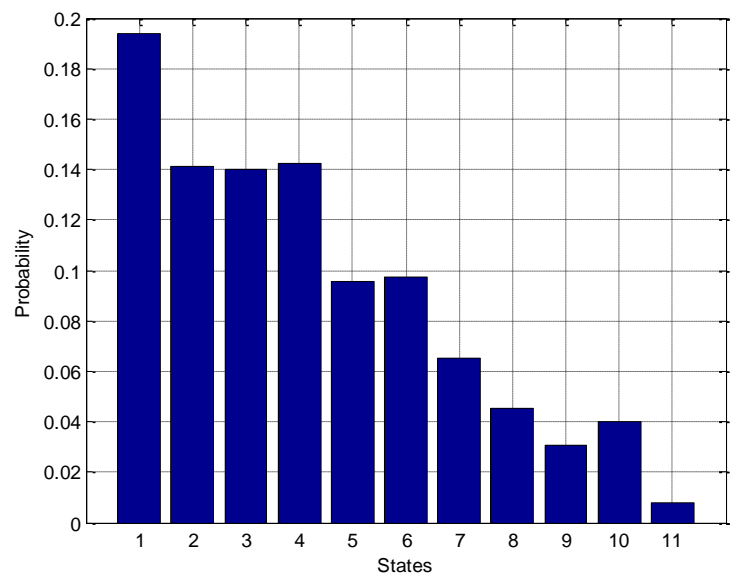

(b) Probability states for $\lambda=5$ packets $/ \mathrm{ms}$,

$$
\begin{aligned}
P_{S S}=0.6816, P_{L S}= & \underset{94.1245,}{ }, P_{s f}=0.9799 \text { and } D_{\text {Mean }}= \\
& 94.3691 \mathrm{~ms}
\end{aligned}
$$

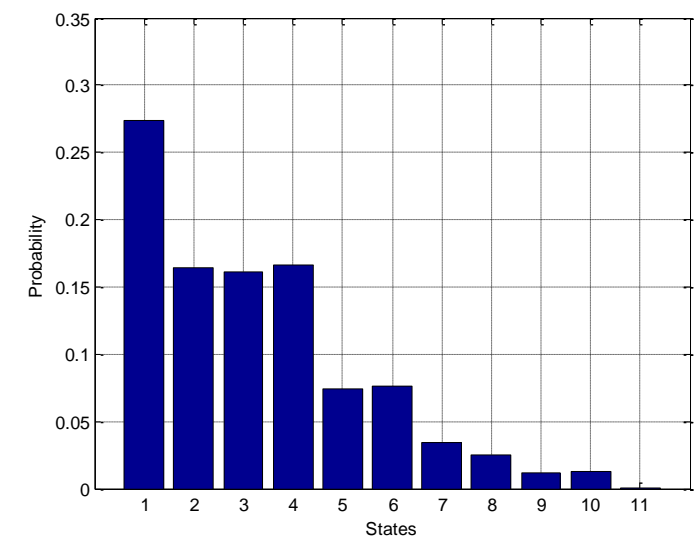

(c) Probability states for $\lambda=10$ packets $/ \mathrm{ms}$, $P_{S S}=0.6771, P_{L S}=0.0496, P_{s f}=0.9625$ and $D_{\text {Mean }}=$ $70.0522 \mathrm{~ms}$

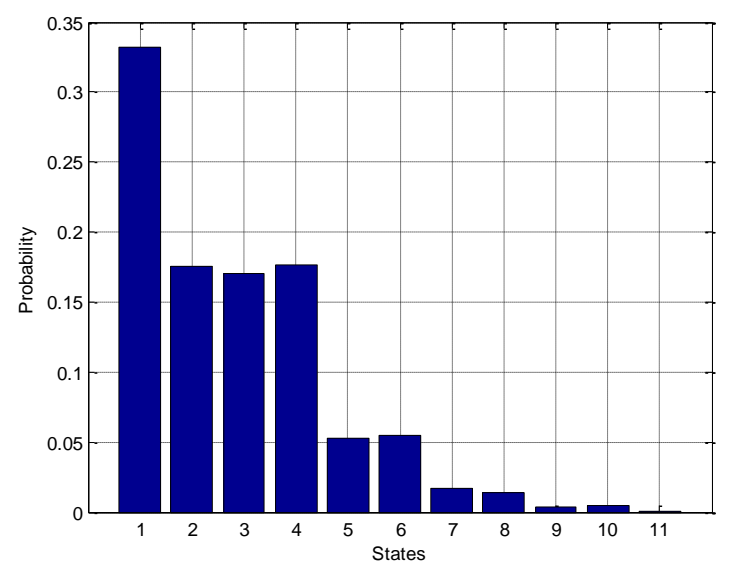

(d) Probability states for $\lambda=15$ packets $/ \mathrm{ms}$, $P_{S S}=0.6458, P_{L S}=0.0216, P_{s f}=0.9463$ and $D_{\text {Mean }}=58.58$ ms 


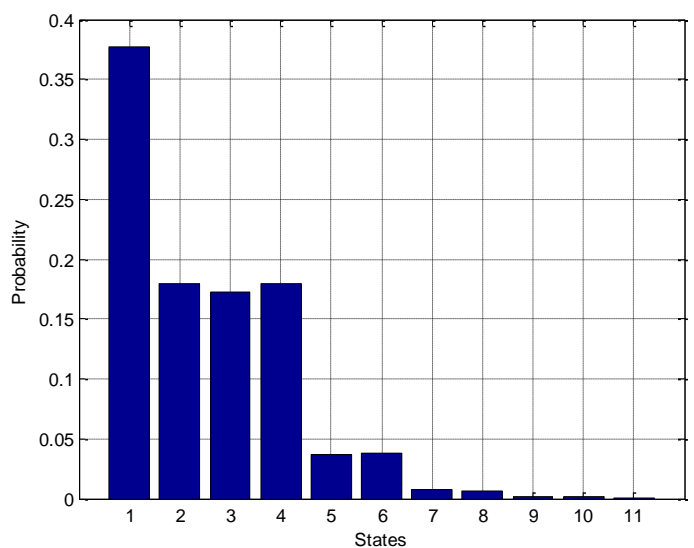

(e) Probability states for $\lambda=20$ packets $/ \mathrm{ms}$,

$$
P_{S S}=\mathbf{0 . 6 1 3 6 ,}, P_{L S}=\underset{52.0882 \mathrm{~ms}}{\mathbf{0 . 0 0 9 4}, P_{s f}=0.9325 \text { and } D_{\text {Mean }}=}
$$

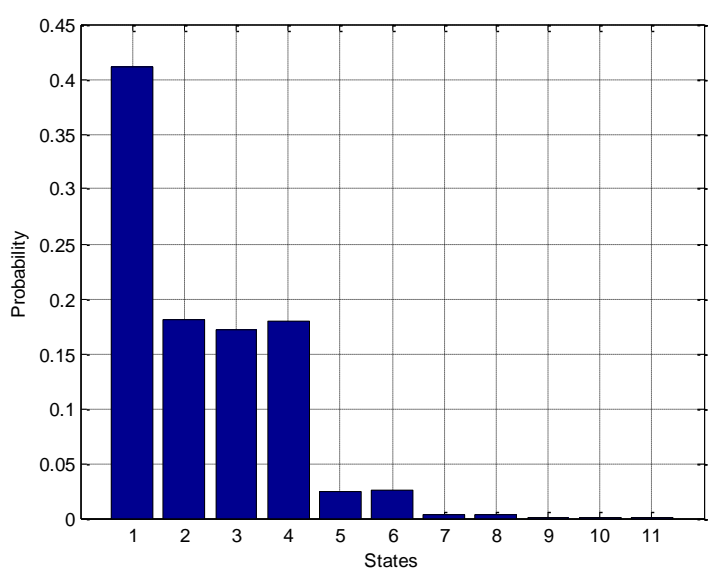

(f) Probability states for $\lambda=25$ packets $/ \mathrm{ms}$,

$$
P_{S S}=0.5850, P_{L S}=0.0040, P_{s f}=0.9213 \text { and } D_{\text {Mean }}=
$$
$48.0923 \mathrm{~ms}$

Figure.2 Probability of 11 states of the state transition chain of Figure.1

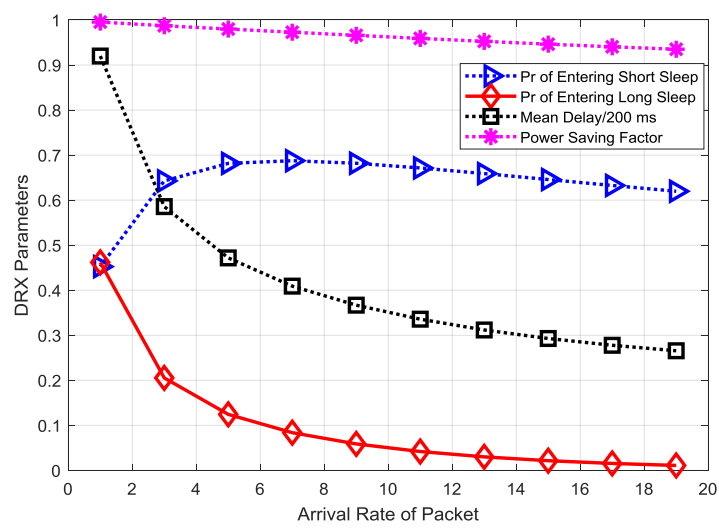

Figure.3 Variation of DRX parameters against the packet arrival rate

Figure. 4 shows the variation of a power-saving factor against packet arrival rate in a session taking $T_{S}$ as a parameter. The power-saving factor decreases exponentially with an increase in packet arrival rate since a higher packet arrival rate will keep the UE busier. With an increase in $T_{S}$ the UE will stay longer duration at short sleep state also visualized from
Figure.4. Al low arrival rate the UE will almost stay in long sleeping hence the impact of $T_{S}$ will be less prominent compared to a moderate or higher value of packet arrival rate found from Figure.4. The impact of $T_{L}$ will be very little on the power saving factor as shown in Figure. 5 since the probability of entering al long sleep is very low.

Next, the variation of mean delay in ms is plotted against the packet arrival rate taking $T_{S}$ as a parameter, where mean delay decreases with an increase in $\lambda$ but increases with an increase in $T_{S}$ shown in Figure.6. For a fixed probability of entering short sleep, the mean waiting time at short sleep will be higher for a larger value of $T_{S}$. The impact of $T_{L}$ is less prominent on mean delay as shown in Figure.7 since the probability of entering at long sleep is low as found in Figure.5. The DRX algorithm saves energy of battery (indicated by a powersaving factor) at the expends of mean delay of buffer since when a new session arrives with several numbers of packets in the midst of sleep state then the packets will be buffered till the start of next DRX cycle. This phenomenon is found in Figure.6. When a UE stays in sleep for long then the energy of its battery is saved found from Figure.4. A 3D plot of arrival rate, mean delay and power-saving factor is shown in Figure.8, which reveals the tradeoff among the three parameters.

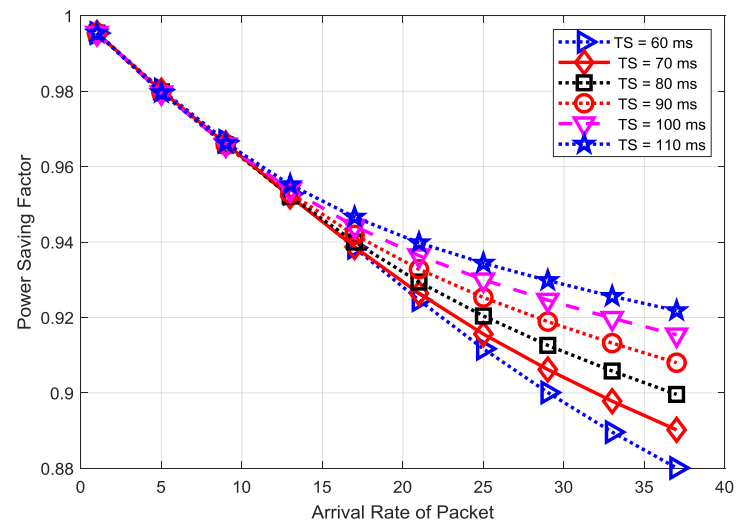

Figure.4 Variation of power-saving factor taking $T_{S}$ as a parameter

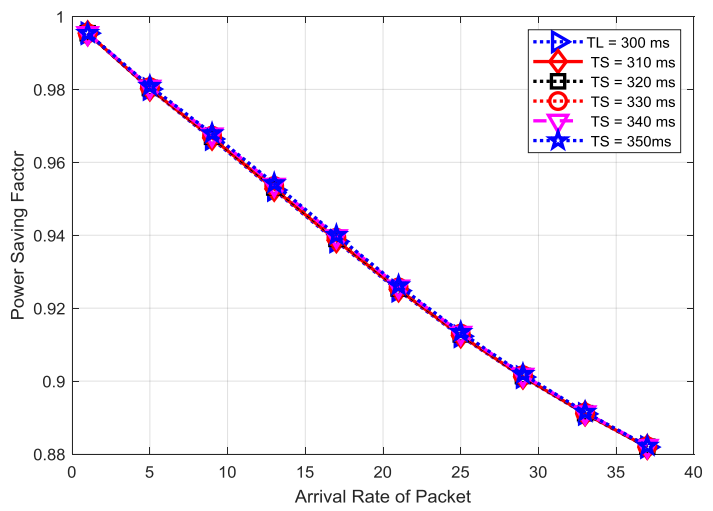

Figure.5 Variation of power-saving factor taking $T_{L}$ as a parameter 


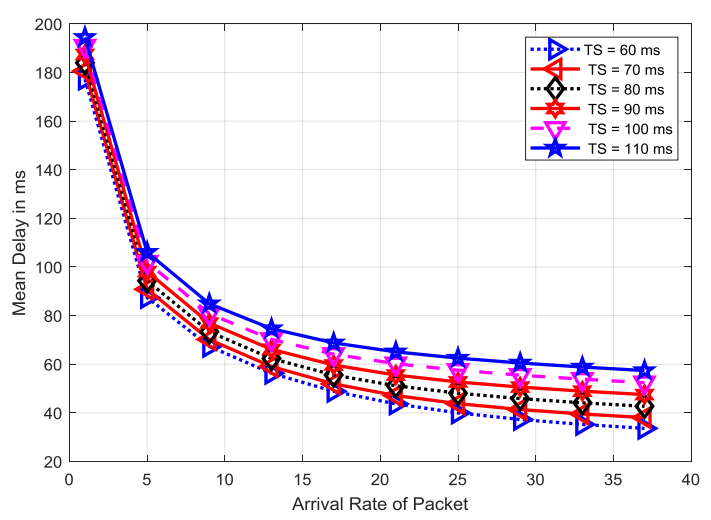

Figure.6 Variation of mean delay factor taking $T_{S}$ as a parameter

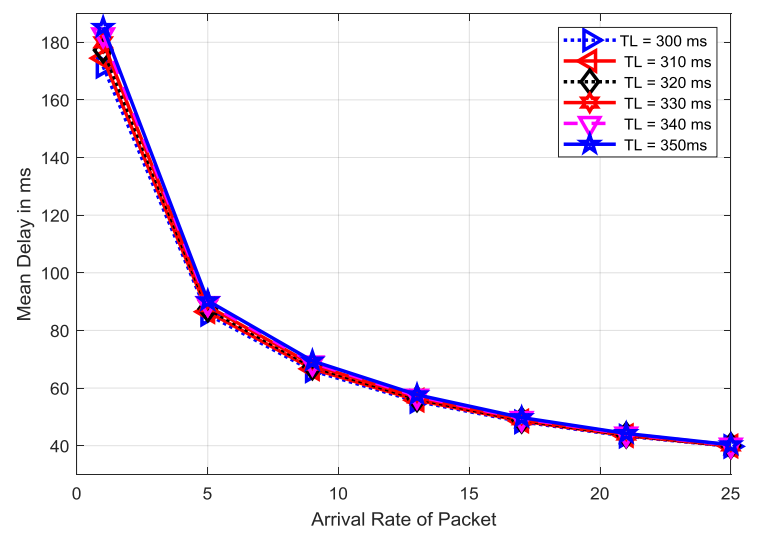

Figure.7 Variation of mean delay factor taking $T_{L}$ as a parameter

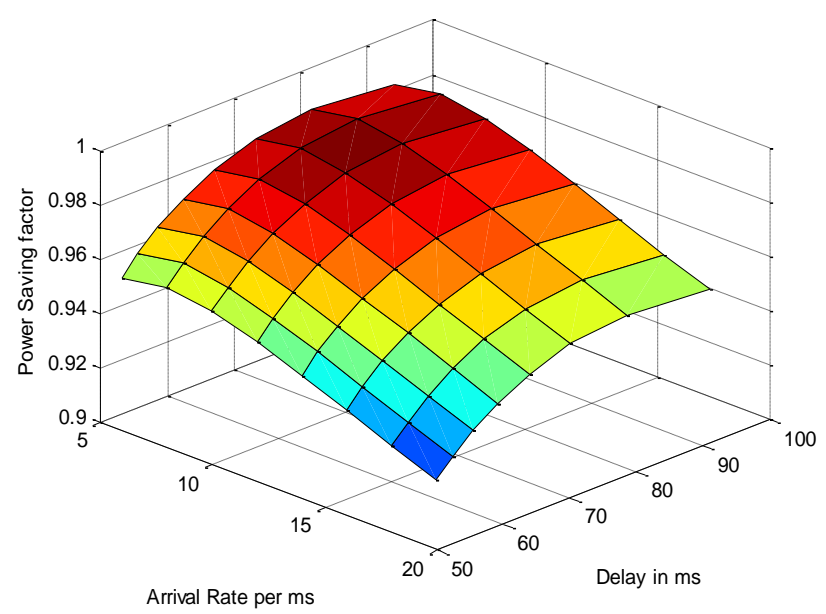

Figure.8 Tradeoff between mean delay and power-saving factor

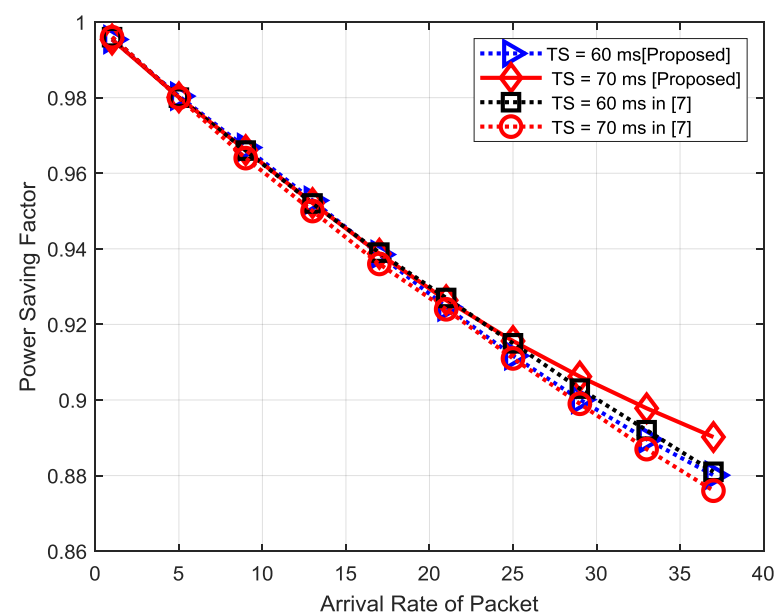

Figure.9 Comparison of the power-saving factor with [7]

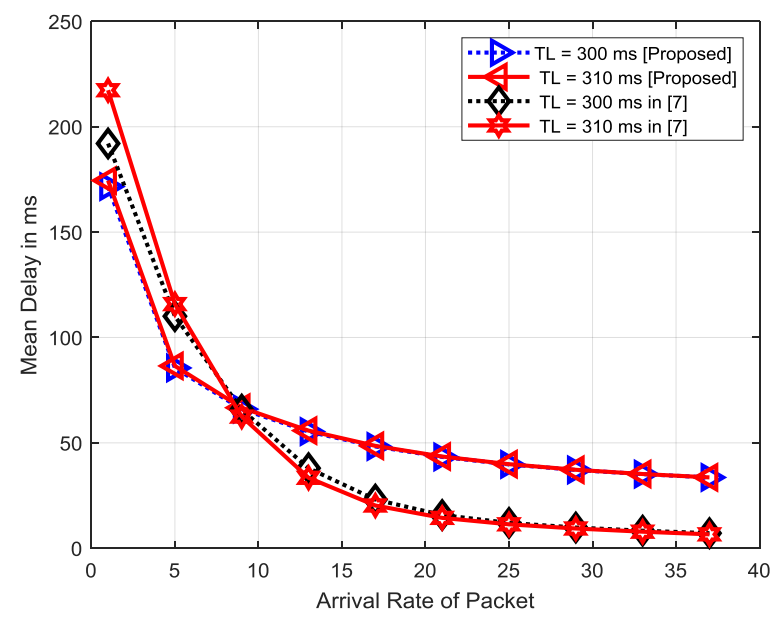

Figure.10 Comparison of mean delay with [7]

Finally, this paper is compared for the power saving factor with [7] for $T_{S}=60 \mathrm{~ms}$ and $70 \mathrm{~ms}$ as shown in Figure. 9. At lower arrival rate $\lambda<25$ packets $/ \mathrm{ms}$, the four $P_{s f}$ of the graph are very closed and all are above $92 \%$. For higher arrival rate $\lambda>25$ packets $/ \mathrm{ms}$, the traffic model used in this paper shows little improvement for $T_{S}=70 \mathrm{~ms}$ but for $T_{S}=60 \mathrm{~ms}$ the previous work reveals the better result. Our proposed model is superior to [7] at higher $T_{S}$. Again, the mean delay for the traffic model of the paper shows better results at low packet arrival rate $\lambda<8$ packets/ms but at higher arrival rate, the traffic model of [7] shows much better results as shown in Figure.10. The main reason for such improvement is: the service time of the packet of [7] follows general distribution instead of exponential service time of this paper. The proposed model of the paper is suitable for an asynchronous connectionless link (ACL) where data integrity is more important than avoiding latency; provided the model of [7] is suitable for synchronous connection-oriented (SCO) link, where avoiding latency is more important than integrity (error-free delivery).

\section{CONCLUSION}

In this paper, the tradeoff between mean delay and powersaving factor are analyzed and the profile of both parameters are shown against the arrival rate of a packet. A comparison is made with previous work and found a better result on mean delay and power-saving factor at lower and higher offered traffic respectively. Still, there are the scopes to analyze the 
DRX (discontinuous reception) for $M / G / 1 / m, M / D / 1 / m$ and $G / G / 1$ traffic model to observe the relative power saving factor and mean delay of TCP/IP, ATM and mixed traffic of the wireless network. Two-dimensional Markov chain can be designed for traffic of dissimilar BW to observe the parameters of DRX under a complete sharing scheme of traffic. In the future, a phase-type Markov renewal process will be designed with active state, short DRX state and long DRX state considering the active state as the absorbing state.

\section{REFERENCES}

[1] Chun-Chuan Yang, Jeng-Yueng Chen, Yi-Ting Mai, and Ching-Hsiang Liang, 'Design of a Load-based DRX Scheme for Non-Real-Time Traffic in LTE,' Proceedings of the International Multi Conference of Engineers and Computer Scientists 2014 vol. II, pp.1-6, IMECS 2014, March 12 - 14, 2014, Hong Kong.

[2] 3GPP TR 36.321, 'Evolved Universal Terrestrial Radio Access (EUTRAN); Medium Access Control (MAC) protocol specification,' ver. 10.5.0, Mar 2012.

[3] Wang, Hwang-Cheng, Chih-Cheng Tseng, Guan-Yun Chen, Fang-Chang Kuo, and Kuo-Chang Ting, 'Accurate analysis of delay and power consumption of LTE DRX mechanism with a combination of short and long cycles,' In Wireless Personal Multimedia Communications (WPMC), 2012 15th International Symposium on, pp. 384-388. IEEE, 2012.

[4] Yen-Wei Kuo and Li-Der Chou, 'Power Saving Scheduling Scheme for Internet of Things over LTE/LTE-Advanced Networks,' Mobile Information Systems, vol. 2015, Article ID 971538, 11 pages, 2015. doi:10.1155/2015/971538.

[5] Fowler, Scott, Ranjeet S. Bhamber, and Abdel hamid Mellouk. 'Analysis of adjustable and fixed DRX mechanism for power saving in LTE/LTE-Advanced,' In Communications (ICC), 2012 IEEE International Conference on, pp. 1964-1969, IEEE, 2012.

[6] Koc, Ali T., Satish C. Jha, Rath Vannithamby, and Murat Torlak, 'Optimizing DRX configuration to improve battery power saving and latency of active mobile applications over LTE-A network,' In Wireless Communications and Networking Conference (WCNC), 2013 IEEE, pp. 568-573, IEEE, 2013.

[7] Sunggeun Jin and Daji Qiao, 'Numerical Analysis of the Power Saving in 3GPP LTE Advanced Wireless Networks,' IEEE Transactions on Vehicular Technology, vol. 61, no. 4, pp. 1779-1785, May 2012.

[8] Tseng, Chih-Cheng, Hwang-Cheng Wang, Fang-Chang Kuo, Kuo-Chang Ting, Hsiao-Hwa Chen, and Guan-Yun Chen, 'Delay and power consumption in LTE/LTE-A DRX mechanism with mixed short and long cycles,' IEEE Transactions on Vehicular Technology, vol. 65, Issue 3, pp.1721-1734, March 2016.

[9] Herrería-Alonso, Sergio, Miguel Rodríguez-Pérez, Manuel Fernández-Veiga, and Cándido López-García, 'Adaptive DRX scheme to improve energy efficiency in LTE networks with bounded delay,' IEEE Journal on Selected Areas in Communications, vol.33, no. 12, pp.2963-2973, Dec' 2015.

[10] Ramazanali, Hawar, and Alexey Vinel, 'Mean queuing delay in LTE DRX,' IEEE Wireless Communications Letters, 5, no. 4, pp. 444-447, aug 2016.
[11] Hawar Ramazanali and Alexey Vinel, 'Tuning of LTE/LTE-A DRX parameters,' 2016 IEEE 21st International Workshop on Computer Aided Modelling and Design of Communication Links and Networks (CAMAD), 23-25 Oct' 2016, Toronto, Canada.

[12] Francois, Frederic, Omer H. Abdelrahman and Erol Gelenbe, 'Towards Assessment of Energy Consumption and Latency of LTE UEs During Signaling Storms,' Information Sciences and Systems 2015, pp. 45-55, Springer International Publishing, 2016.

[13] Mohammad Asif Hossain, Mohammad Imdadul Islam and M R Amin, 'Load Sensitive Power Saving Technique for 4G Mobile Network under Limited User Traffic,' Communications and Network, vol. 8, no. 02, pp.79-87, 2016.

[14] Mohammad Asif Hossain, Md. Imdadul Islam, and M R Amin, 'Analysis of Sleep Window of IEEE802. $16 \mathrm{~m}$ Network Based on State Transition Chain,' Journal of Computer and Communications, vol.3, no. 12, pp. 77-83, 2015.

[15] Mohammad Asif Hossain, Md. Imdadul Islam and M.R. Amin, 'Analytical Modelling of DRX Mechanism to Enhance the Energy Efficiency in LTE,' 2016 Asia Multi Conference on Modelling and Simulation, IEEE, pp. 6973, Kota Kinabalu, Sabah, Malaysia, 2016.

[16] Hui-Ling Chang and Meng-Hsun Tsai, 'Optimistic DRX for Machine-Type Communications in LTE-A Network,' IEEE Access, vol. 6, pp. 9887 - 9897, January 2018.

[17] Mukesh Kumar Maheshwari, Abhishek Roy , and Navrati Saxena, ' DRX over LAA-LTE-A New Design and Analysis Based on Semi-Markov Model,' IEEE Transactions on Mobile Computing, vol. 18, no. 2, pp.276-289, February 2019.

[18] Mads Lauridsen, Daniela Laselva, Frank Frederiksen and Jorma Kaikkonen, '5G New Radio User Equipment Power Modeling and Potential Energy Savings,' 2019 IEEE 90th Vehicular Technology Conference (VTC2019-Fall), 22-25 Sept. 2019, Honolulu, HI, USA.

[19] Md. Imdadul Islam, Md. Fazlay Rabbi, Risala Tasin Khan and Jesmin Akhter, 'Performance Evaluation of Cognitive Radio Network Based on 2-D Markov Chain,' Journal of Telecommunications and Information Technology (JTIT), pp.39-44, vol.3, 2015.

[20] Dulal Chakraborty, Abul Kalam Azad, Md. Imdadul Islam and A S M Rahaman, 'Performance Analysis of CSMA/CA Network using Modified State Transition Chain,' International Journal of Computer Science and Information Security, vol. 14, no. 12, pp.1064-1070, DEC 2016.

[21] Scott Fowler, Ahmed Omar Shahidullah, Mohammed Osman, Johan M. Karlsson and Di Yuan 'Analytical evaluation of extended DRX with additional active cycles for light traffic' Computer Networks(Elsevier), vol. 77, pp. 90-102, February 2015.

[22] Arpit Banker and B. Rebekka, 'Efficient Optimization of Wake Up Delay and Power Saving of Adjustable DRX Mechanism In LTE Network,' Proceedings of the 2nd International Conference on Trends in Electronics and Informatics (ICOEI 2018), pp.765-771, 11-12 May 2018, Tirunelveli, India. 


\section{AUTHORS' PROFILE}

Mohammad Asif Hossain received his B.Sc. degree in information and communications engineering, M.Sc. degree in telecommunications engineering, and MBA degree in marketing from East West University (EWU), Dhaka, Bangladesh, in 2006, 2007, and 2011, respectively. He is currently pursuing a Ph.D. degree in computer science at the University of Malaya in Malaysia. He was an assistant professor at the Department of Electronics and Communications Engineering at East West University, Dhaka, Bangladesh. He is currently working as a graduate research assistant at the University of Malaya. His research interests include cognitive radio, vehicular networks, wireless networking, machine learning, energy harvesting and SWIPT. $\mathrm{He}$ was a recipient of Chancellor's Gold Medals for his excellent performance in his B.Sc. and M.Sc. degrees.

Md. Imdadul Islam had completed his B.Sc. and M.Sc Engineering in Electrical and Electronic Engineering from Bangladesh University of Engineering and Technology, Dhaka, Bangladesh in 1993 and 1998 respectively and had completed his Ph.D. degree from the Department of Computer Science and Engineering, Jahangirnagar University, Dhaka, Bangladesh in the field of network traffic in 2010. He is now working as a Professor at the Department of Computer Science and Engineering, Jahangirnagar University, Savar, Dhaka, Bangladesh. Previously, he worked as an Assistant Engineer in Sheba Telecom (Pvt.) LTD (A joint venture company between Bangladesh and Malaysia, for Mobile cellular and WLL), from Sept.1994 to July 1996. Dr. Islam has very good field experience in the installation and design of the mobile cellular network, Radio Base Stations and Switching Centers for both mobile and WLL. His research field is network traffic, wireless communications, wavelet transform, OFDMA, WCDMA, adaptive filter theory, array antenna systems, ANFIS and Machine Learning. He has more than a hundred and eighty research papers in national and international journals and conference proceedings.

Fahima Tabassum has completed her B.Sc. (Hons.) from the Department of Computer Science and Engineering, Jahangirnagar University, Savar, Dhaka, Bangladesh in 2003 and M.S from the same department in 2010. She currently is pursuing her Ph.D. degree in the same department. She has a number of publications in different reputed journals. She is also working as an associate professor at the Institute of Information Technology, Jahangirnagar University, Savar, Dhaka 1342, Bangladesh.

M. R. Amin received his B.S. and M.S. degrees in Physics from Jahangirnagar Uni-versity, Dhaka, Bangladesh in 1984 and 1986 respectively and his Ph.D. degree in Plas-ma Physics from the University of St. Andrews, U. K. in 1990. $\mathrm{He}$ is a Professor of Department of Mathematical and Physical Sciences at East West University, Dhaka, Bangladesh. He served as a Post-Doctoral Research Associate in Electrical Engineering at the University of Alberta, Canada, during 1991-1993. He was an Alexander von Humboldt Research Fellow at the Max-Planck Institute for Extraterrestrial Physics at Garching/Munich, Germany during 1997-1999. His current research fields are wireless communications and networks and also nonlinear plasma dynamics. He is a member of the IEEE. 